\title{
Case \\ Report \\ A $t(X ; 18)$ SYT-SSX2 positive synovial sarcoma in the pelvis of a young adult male: A rare case report with review of literature
}

\author{
Rekhi B' ${ }^{1}$, Jambhekar NA ${ }^{1}$, Desai SB ${ }^{1}$, Basak R ${ }^{2}$, Puri A ${ }^{3}$, Agrawal $\mathbf{M}^{3}$ \\ ${ }^{1}$ Department of Pathology, Tata Memorial Center, Mumbai; '2Department of Molecular Pathology, \\ Advanced Centre for Treatment, Research and Education in Cancer (ACTREC), Khargar, Navi Mumbai; \\ ${ }^{3}$ Department of Orthopedic Surgical Oncology, Tata Memorial Center, Mumbai, India.
}

Correspondence to: Dr. Bharat Rekhi, E-mail: rekhi.bharat@gmail.com

\begin{abstract}
Synovial sarcoma is uncommonly documented in the pelvis. Rarely, such cases have dealt with molecular analysis. A 19-year-old boy presented with pain and swelling in his left lower limb of two months duration. He developed acute urinary retention four days prior to his hospital admission, wherein radiological examination unraveled a large soft tissue mass, displacing his pelvic muscles, along with a lytic lesion involving his right pubic bone. Biopsy showed a cellular spindle cell sarcoma, exhibiting hemangiopericytoma-like vascular pattern with focal necrosis. Immunohistochemistry (IHC) showed positivity for vimentin, BCL-2, calponin and MIC 2. Cytokeratin (CK) and epithelial membrane antigen (EMA) were negative. MIB 1 count was 70\% (high). P53 was positive. Diagnosis of a poorly differentiated synovial sarcoma was offered and confirmed with a positive $t(X ; 18)$ SYT-SSX2 translocation. This case highlights the value of molecular analysis in diagnosis of a synovial sarcoma at rare sites, especially when IHC results are equivocal and the biopsy material is limited.
\end{abstract}

Key words: Molecular analysis, pelvic synovial sarcoma, pelvic tumors, soft tissue sarcomas, $t(X ; 18)$ SYT-SSX2 translocation.

\section{Introduction}

Synovial sarcoma (SS) is defined as a mesenchymal spindle cell tumor, displaying variable epithelial differentiation and is characterized by a specific chromosomal translocation $\mathrm{t}(\mathrm{X}: 18)$ (p11; q11). ${ }^{[1]}$ It is uncommon, accounts for $5-10 \%$ of soft tissue sarcomas and is unrelated to the synovium. Traditionally, extremities form the commonest sites of its occurrence in 80-90\% cases. $^{[2]} \mathrm{H}$ owever, with the advent of ancillary techniques, it has been identified at unusual locations like head and neck region, lung, prostate. ${ }^{[3-4]}$ Few cases of SS have been documented in the pelvis, especially involving the bone ${ }^{[5-8]}$ Still rare is its objective identification with molecular results in this location. ${ }^{[7]} \mathrm{H}$ erein, we report a t $(X ; 18)$ SYT-SSX2 positive, poorly differentiated synovial sarcoma in the pelvis of a young adult male, describing the value of molecular analysis in ascertaining this diagnosis.

\section{Case Report}

A 19-year-old boy presented with swelling and pain in his left lower limb of two months duration. Four days prior to his hospital admission, he developed acute urine retention. Subsequently, he underwent radiological evaluation.

X-ray pelvis showed a permeative lesion involving his right pubic bone, superior and inferior pubic ramus, including a transverse fracture. In addition, a soft tissue mass was seen displacing the obturator and psoas fat planes.

U Itra fast slice plain and contrast enhanced computed tomography (CT) scan showed a highly vascular, large, lobulated, mixed density mass measuring $13.3 \times 12.4 \times 9.9$. $\mathrm{cm}$ with speck of calcification, in the right inferolateral aspect of urinary bladder, extending 
into the upper thigh. It was seen destroying the pubic bones and the anterior half of right acetabulum. Multiple non-enhancing areas, within the lesion, suggestive of cystic and necrotic components, were noted.

Anteriorly, the lesion extended till the pubic symphisis and posteriorly, up to the anterior surface of sacrum [Figure 1]. All the visceral organs were normal. A diagnosis of 'Ewing' sarcoma was suggested.

Subsequently, a core needle biopsy was performed and the tissue was submitted for histopathological diagnosis.

\section{Histopathological findings}

Grossly, multiple small grey white soft tissue bits aggregating to $1.5 \times 1 \times 0.4 \mathrm{~cm}$ were received and processed for paraffin blocks. $5 \mu$ thick sections were stained with hematoxylin and eosin $(H \& E)$ stain and further subjected to immunohistochemistry (IHC) using Avidin-biotin method. A wide panel of antibody markers was performed viz. vimentin, cytokeratin (CK), cytokeratin ( $A E 1 / A E 3)$, epithelial membrane antigen (EMA), BCL-2, calponin, MIC2, synaptophysin, P53, MIB1, desmin, CD 34, smooth muscle actin (SM A), S-100, C-KIT. (D ako, Produkionsveg, Glostrup, Denmark)

$\mathrm{H}$ and $\mathrm{E}$ stained sections showed a cellular tumor comprising oval to spindly cells, displaying 'staghorn' arrangement of vasculature, reminiscent of a hemangiopericytoma-like (H PC) pattern. Focal areas showed myxoid change, necrosis and apoptosis. Mitoses were $7 / 10$ high power field (hpf). Reticulin stain highlighted HPC pattern and reticulin deposition around single and cell groups along with focal reticulin low

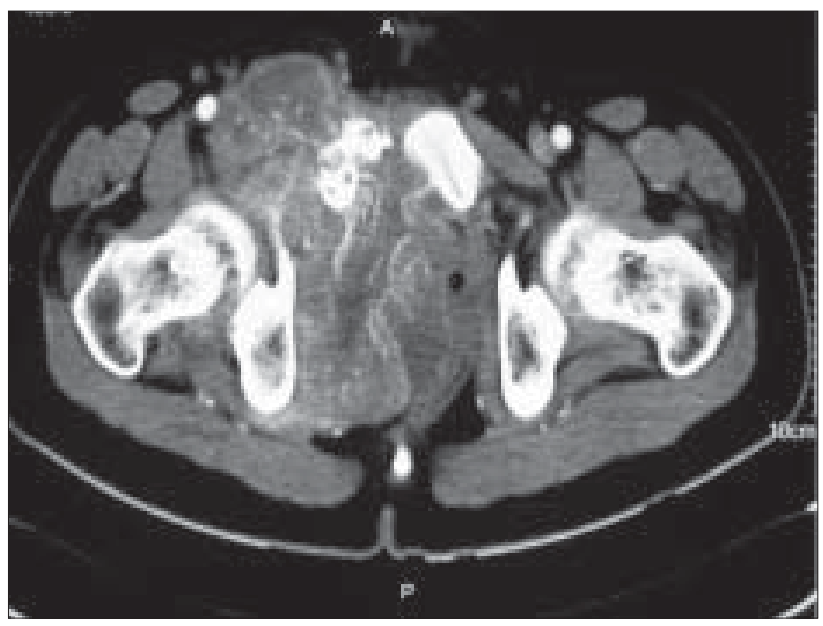

Figure 1: Computed tomography scan abdomen and pelvis showing a large, lobulated, mixed density mass measuring $13.3 \times 12.4 \times 9.9$. $\mathrm{cms}$, destroying the superior pubic ramus, right pubis, anterior half of right acetabulum and inferior half of pubic ramus areas (inset). A provisional diagnosis of a high grade, poorly differentiated synovial sarcoma of spindle cell type was formed [Figure 2 A-D ]

IHC showed diffuse positivity for vimentin, bcl-2, calponin and negativity for cytokeratin (CK), EMA, AE1/AE3, S100, synaptophysin, desmin, SMA, CKIT and $C D 34$, the latter most that highlighted the vascular pattern. MIB 1 was 70\% (high). P53 was positive. MIC 2 showed weak, focal positivity [Figure 3A-F] A diagnosis of a high grade, poorly differentiated, synovial sarcoma of spindle cell type was offered.

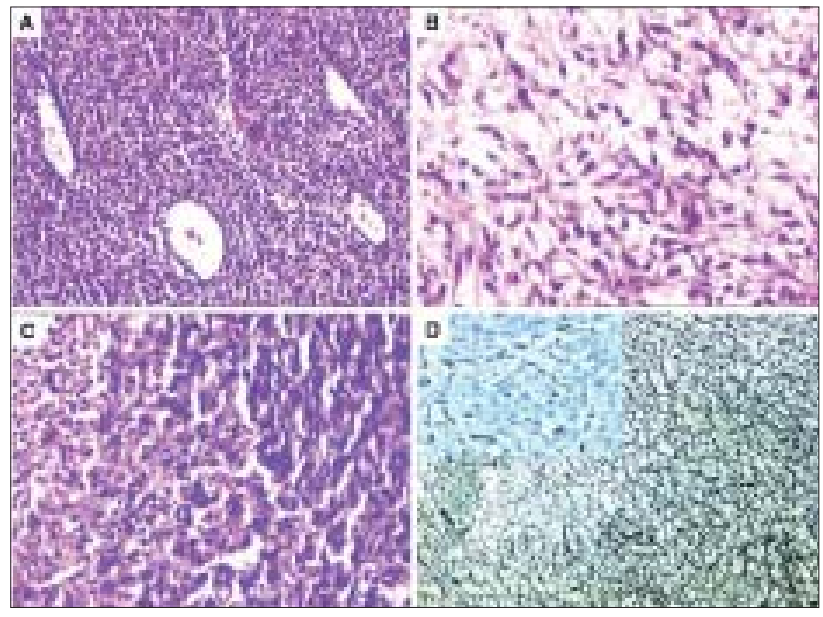

Figure 2: Pathological findings of a pelvic synovial sarcoma. A. A cellular spindle cell tumor showing slit like, gaping vessels reminiscent of a hemagiopericytoma-like pattern. H\&E, x200. Focal areas showing myxoid degeneration. H\&E, x400. C. Areas of necrosis and apoptosis amidst tumor. H\&E, x200. D. Reticulin staining displays increase in reticulin around single cells. Reticulin stain. H\&E, x200. Inset showing areas of low reticulin. Reticulin stain. H\&E, x400.

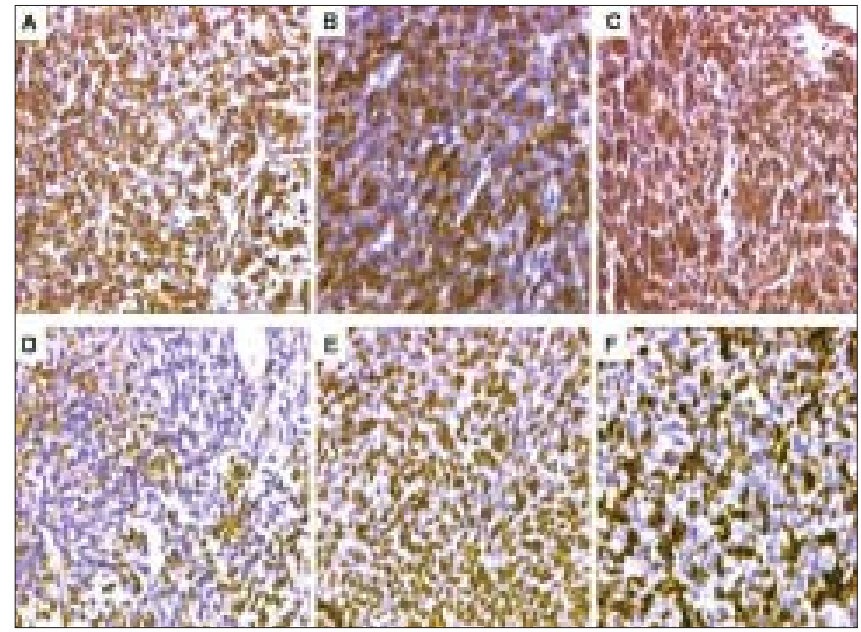

Figure 3: Immunohistochemical (IHC) profile. A. Tumor cells displaying diffuse, strong, vimentin expression. DAB, x200. B. Strong, diffuse BCL-2 positivity in the tumor cells. DAB, x200. C. Strong, diffuse calponin positivity in the tumor cells. DAB, x200. D. Focal MIC-2 positivity in the tumor cells. DAB, x200. E. Intense MIB-1 staining. DAB, x200. F. p53 positivity in the tumor cells. DAB, $\times 200$ 


\section{Molecular analysis}

Further, paraffin block was subjected to RT-PCR analysis. Total RNA was isolated using Optimum ${ }^{T M}$ FFPE RNA Isolation kit (Ambion Diagnostics). This was reverse transcribed using CDNA synthesis kit (Gibco-BRL). The primer sequences were as follows; ${ }^{[7]}$ SYT (Forward): 5' CCA GCA GAG GCC TTA TGG ATA 3'

SSX (Reverse): 5' TTT GTG GGC CAG ATG CTT C $3^{\prime}$

For SYT-SSX translocation detection, PCR was done using following primers;

SYT (Forward): 5' CAA CAG CAA GAT GCA TAC CA 3'

SSX 1 (Reverse): 5' GGT GCA GTT GTT TCC CAT

CG 3'

SSX2 (Reverse): 5' GGC ACA GCT CTT TCC CAT CA 3'

The PCR products were analyzed on $10 \%$ Polyacrylamide gel, which showed a positive band for $\mathrm{t}(\mathrm{X} ; 18)$ SYT-SSX2 translocation. Diagnosis of synovial sarcoma was confirmed [Figure 4].

The patient was a candidate for surgery with adjuvant $\mathrm{CT}$. H owever, unfortunately, he was lost for treatment.

\section{Discussion}

Synovial sarcoma (SS) forms a distinct clinical, morphological and a genetic type of a soft tissue sarcoma, which has been described in various body sites. ${ }^{[3-5]}$ Intraabdominal primary SS is unusual. N early 50 cases have been documented so far. Still rare, is a pelvic location, wherein only five cases have been identified to the best of our knowledge. ${ }^{[5-8]}$ Only one such case has dealt with molecular analysis. ${ }^{[7]}$

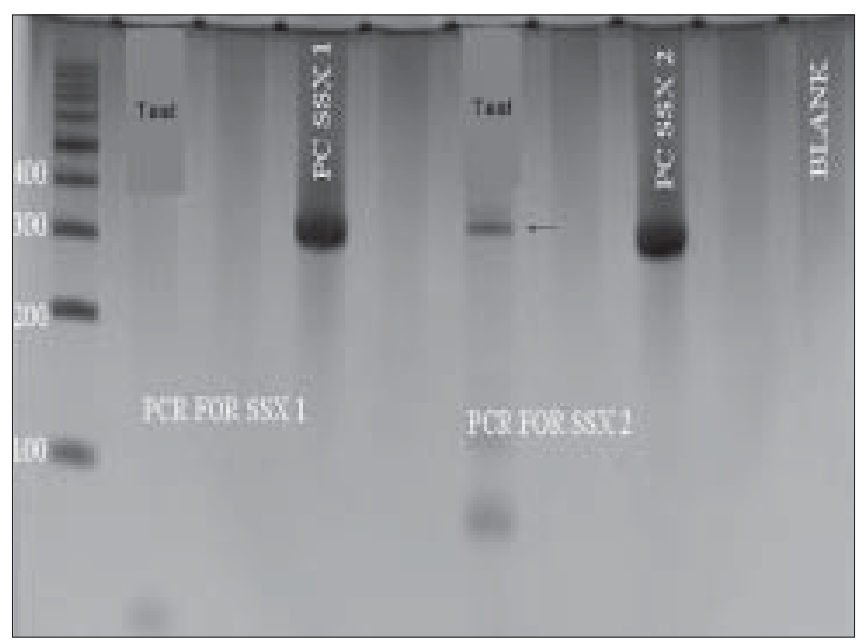

Figure 4: RT-PCR analysis showed positivity for $t(X ; 18)$ SYT-SSX2 translocation
The present case of a SS was seen in a young boy, whose radiological evaluation unraveled a large, bonedestructive pelvic mass, a feature that has not been seen with the similar documented cases. M oreover, he constitutes the youngest of all such cases, wherein the age ranged from 25-72 years ${ }^{[6-8]}$ [Table 1].

Radiologically, a pelvic SS reveals an admixture of cystic and necrotic elements, leading to a "bowl of fruit" sign, as seen in the present case. ${ }^{[8]}$ This led to a putative diagnosis of a Ewing's sarcoma. Biopsy showed a high grade, spindle cell sarcoma, which led to a range of differential diagnoses, including a malignant peripheral nerve sheath tumor (MPNST), Ewing sarcoma, a leiomyosarcoma (LMS), a gastrointestinal stromal tumor (GIST), a desmoplastic small round cell tumor (DSRCT) and a sarcomatoid mesothelioma, as noted in earlier reports. ${ }^{[7,8]}$ Despite an X-ray appearance of a permeative lesion and cytomorphological presence of oval cells, conspicuous presence of short spindly cells, made diagnosis of Ewing sarcoma, less likely. The presence of myxoid areas led to MPNST, LMS and GIST as other differentials. While lack of wavy/ 'serpentine' nuclei was helpful in ruling out an M PNST; lack of 'cigar-shaped' nuclei with blunt ends made diagnosis of a LMS, less likely. N egative S-100, desmin and SMA expression helped in ruling out these possibilities. GIST and DSRCT were included as other differentials, as seen earlier. ${ }^{[7]}$ Lack of epithelial markers ( $C K, E M A$ and $A E 1 / A E 3$ ), made DSRCT as a less likely possibility. Lack of epithelial marker expression in a SS was also noted by Cole et al[ ${ }^{[7]}$ but this finding was in contrast to other studies. ${ }^{[6,7]}$ In a series of 20 cases of poorly differentiated SS, van de Rijn $\mathrm{M}^{[9]}$ identified EM A positivity in $92 \%$ cases and CK in $42 \%$ cases. This explains a rare IHC profile in our case. At the same time, there is a possibility that negative epithelial marker expression might have been due to limited biopsy material in our case. N onetheless, BCL 2 and calponin positivity with focal MIC-2 expression helped to reinforce this diagnosis, as noted by others. ${ }^{[9-11]}$ GIST was ruled out in view of CKIT and CD 34 negativity. ${ }^{[10]}$

In view of equivocal IHC results, molecular analysis was recommended, which showed $t(X ; 18)$ SYT-SSX2 positive transcript. SS has been shown to consistently express a $\mathrm{t}(\mathrm{X} ; 18 ; \mathrm{p} \mathrm{11;q11)}$ translocation, which usually represents either of the 2 gene fusions, SYTSSX 1 or SYT-SSX 2 that encode specific putative transcriptional proteins. ${ }^{[12,13]}$ A part for its diagnostic value, this translocation was believed to have a prognostic value. Compared to SYT-SSX2 fusion, mostly seen in monophasic SS, a SYT-SSX1 positive transcript, more commonly seen in a biphasic SS, was associated with a relatively unfavorable prognosis. ${ }^{[14]} \mathrm{H}$ owever, 
Rekhi B, et al.: $\mathrm{t}(\mathrm{X} ; 18)$ SYT-SSX2 positive pelvic synovial sarcoma

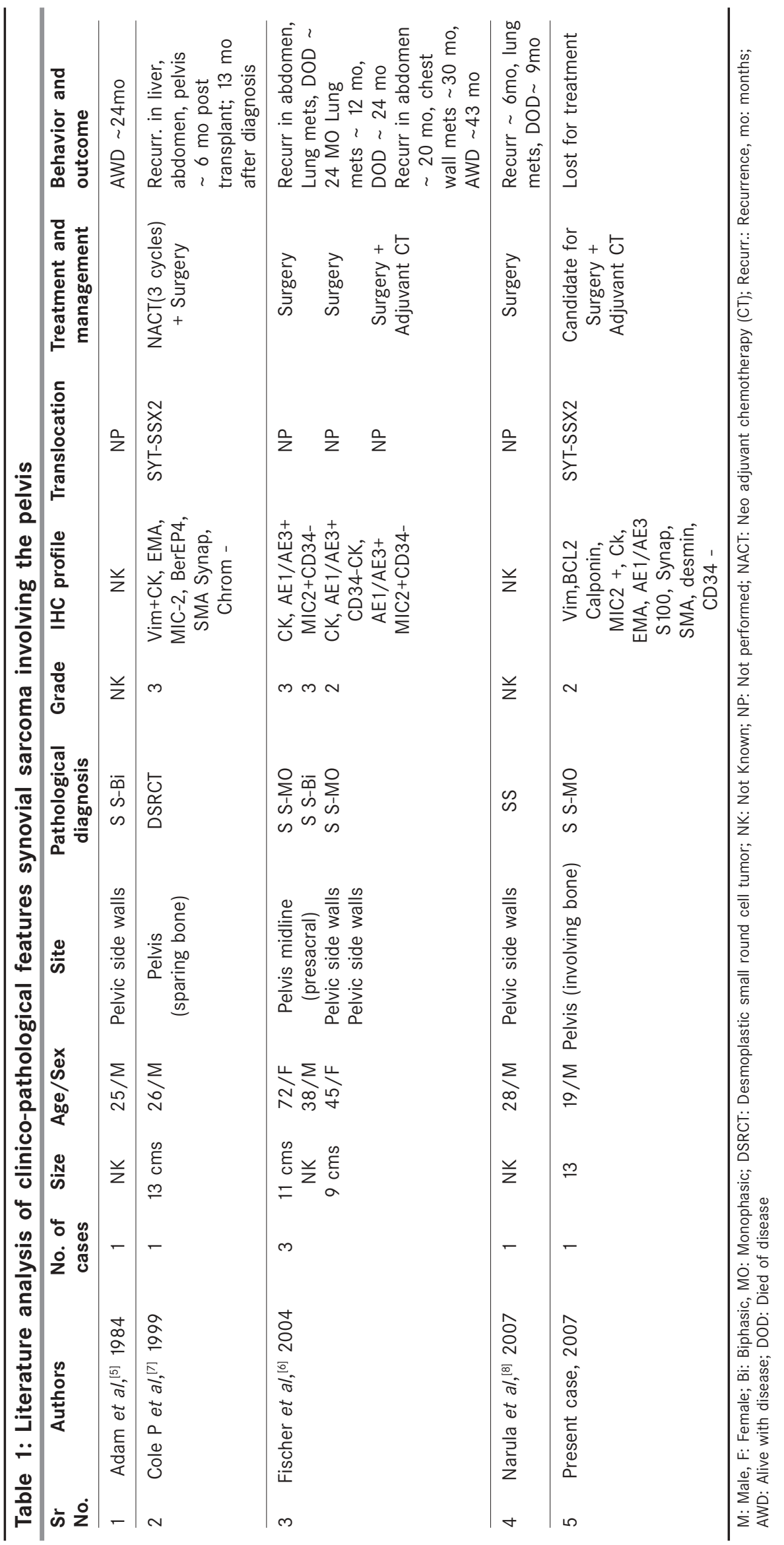


lately studies, including the one by Guillou et al[15] have observed lesser prognostic value of specific transcript in comparison to tumor grade.

Even though, the present case of a pelvic SS of spindle cell type, like the one described by Cole et al[ ${ }^{[7]}$ exhibited similar SYT-SSX2 transcript, the other parameters forecast an unfavorable prognosis. A part from site, larger tumor size, poor differentiation, necrosis, apoptosis along with high mitoses are indicators of a grim prognosis, as noted earlier. ${ }^{[1,6,7,16]}$ As per literature, it has been seen that invariably, pelvic SSs are associated with a dismal outcome as a result of local recurrences and metastasis, latter that has been noted in $4 / 6$ similar documented cases. ${ }^{[6-8]}$

Surgery is the treatment mainstay. H owever, marginal clearance is difficult to achieve in this location. Adjuvant chemotherapy (CT) and radiotherapy (RT) have been given ${ }^{[6,8,16]}$ Despite that, recurrences and metastasis have been noted. O ur case is a candidate for surgery with adjuvant CT. In a nutshell, this case reinforces value of molecular analysis in a SS at uncommon sites.

\section{References}

1. Fisher C, Bruijn DRH, Geurts van Kessel A. In: Tumors of soft tissue and bone. Pathology and genetics. World Health Organization classification of tumors. Lyon: IARC Press; 2002.

2. Weiss SV, Goldblum R, editors. Enzinger and Weiss's soft tissue tumours, $4^{\text {th }}$ ed, St Louis: CV Mosby; 2001.

3. Tilakaratne WM. Synovial sarcoma of the mandible. J Oral Pathol Med 2006;35:61-3.

4. Fritsch M, Epstein JI, Perlman EJ, Watts JC, Argani P: Molecularly confirmed primary prostatic synovial sarcoma. Hum Pathol 2000; 31: $246-250$
5. Adam YG, Oland J, Halevy A, Park SH, Ayala AG, Ro JY. Primary retroperitoneal soft-tissue sarcomas. J Surg Oncol 1984;25:8-11.

6. Fisher C, Folpe AL, Hashimoto H, Weiss SW. Intra-abdominal synovial sarcoma: A clinicopathological study. Histopathology 2004;45:245-53

7. Cole P, Ladanyi M, Gerald WL, Cheung NK, Kramer K, LaQuaglia MP, et al. Synovial sarcoma mimicking desmoplastic small roundcell tumor: Critical role for molecular diagnosis. Med Pediatr Oncol 1999;32:97-101.

8. Narula MK, Madan R, Pathania OP. Primary intra-abdominal synovial sarcoma. Appl Radiol 2007;36:48A.

9. van de Rijn M, Barr FG, Xiong QB, Hedges M, Shipley J, Fisher C. Poorly differentiated synovial sarcoma: An analysis of clinical, pathologic, and molecular genetic features. Am J Surg Pathol 1999;23:106-12.

10. Suster S, Fisher C, Moran CA. Expression of bcl-2 oncoprotein in benign and malignant spindle cell tumors of soft tissue, skin, serosal surfaces and gastrointestinal tract. Am J Surg Pathol 1998;22:863-72.

11. Fisher C, Montgomery E, Healy V. Calponin and h-caldesmon expression in synovial sarcoma: The use of calponin in diagnosis. Histopathology 2003;42:588-93.

12. Shipley JM, Clark J, Crew AJ, Birdsall S, Rocques PJ, Gill S, et al. The $t(X ; 18)(p 11.2 ; q 11.2)$ translocation found in human synovial sarcomas involves two distinct loci on the X chromosome. Oncogene 1994;9: 1447-53.

13. Ladanyi M: The emerging molecular genetics of sarcoma translocations. Diagn Mol Pathol 1995;4:162-73.

14. Ladanyi M, Antonescu CR, Leung DH, Woodruff JM, Kawai A, Healey $\mathrm{JH}$, et al. Impact of SYT-SSX fusion type on the clinical behavior of synovial sarcoma: A multi-institutional retrospective study of 243 patients. Cancer Res 2002;62:135-40.

15. Guillou L, Benhattar J, Bonichon F, Gallagher G, Terrier P, Stauffer E, et al. Histologic grade, but not SYT-SSX fusion type, is an important prognostic factor in patients with synovial sarcoma: A multicenter, retrospective analysis. J Clin Oncol 2004;22:4040-50.

16. Brodsky JT, Burt ME, Hajdu SI, Casper ES, Brennan MF. Tendosynovial sarcoma. Clinicopathologic features, treatment, and prognosis. Cancer 1992;70:484-9.

Source of Support: Nil, Conflict of Interest: None declared.

\section{Author Help: Sending a revised article}

1) Include the referees' remarks and point to point clarification to those remarks at the beginning in the revised article file itself. In addition, mark the changes as underlined or coloured text in the article. Please include in a single file

a. referees' comments

b. point to point clarifications on the comments

c. revised article with text highlighting the changes done

2) Include the original comments of the review ers/editor with point to point reply at the beginning of the article in the 'Article File'. To ensure that the reviewer can assess the revised paper in timely fashion, please reply to the comments of the referees/editors in the follow ing manner.

- There is no data on follow-up of these patients.

Authors' Reply: The follow up of patients have been included in the results section [Page 3, para 2]

- Authors should highlight the relation of complication to duration of diabetes.

Authors' Reply: The complications as seen in our study group has been included in the results section [Page 4, Table] 